\title{
EDITORIAL
}

\section{"Occupational Asthma": A matter of concern}

\author{
C.E. Mapp*
}

An association between asthma and work was first recognized by Hippocrates, who described its presence in metal workers, tailors, fullers, horsemen, farmhands and fishermen. In 1713, in his "De morbis artificum diatriba" Bernardino Ramazzini provided a description of diseases due to various occupations, and emphasized the necessity for a physician who sees a patient to put the following questions: "In what trade do you work?" and "What other occupations have you had?"

The complex relationship between type of occupational sensitizer, intensity of exposure, individual predisposing factors and development of occupational asthma is still not fully understood. The last 10 yrs have seen widespread efforts to clarify the mechanisms involved in occupational asthma. Indeed, asthma is now the most frequent occupational lung disease in industrialized countries. This is the reason for the collaborative interest of allergists, immunologists, pulmonologists, occupational health specialists and epidemiologists.

The orientation of this present series entitled "Occupational Asthma" reflects the goal of an international effort to review the new advances in this field. A. Cartier reviews the current criteria used in the definition and diagnosis of occupational asthma. He discusses the different steps of investigation, which are history, pulmonary function tests, immunological tests, "peak expiratory flow", nonspecific airway responsiveness monitoring and specific inhalation challenge. He emphasizes that a negative inhalation challenge does not absolutely rule out the diagnosis of occupational asthma in a worker who has not been exposed to work for several months.

W.S. Beckett stresses the important role of systematic epidemiological approaches to yield new insights into the clinical characteristics of occupational asthma. He also emphasizes that, although occupational asthma is a relatively common illness, very little is known about quantitative exposure-response relationships.

The role of aetiological causes of occupational asthma is reviewed by M. Chan Yeung and J.L. Malo. They stress that the absence of the suspected agent in any list of aetiological causes of occupational asthma should not exclude the diagnosis. They conclude that an international effort to prepare a data base, similar to the Minitel System in France, would be helpful to clinicians and researchers in this field.

My colleagues and I review the current knowledge about the mechanisms and pathology of occupational asthma. Although more than one mechanism may be involved in the disease, we suggest that immunological mechanisms and airway inflammation play an important role. We also report on the possible role of genetic mechanisms in occupational asthma.

M.H. Karol discusses the advantages and disadvantages of animal models of occupational asthma, and suggests that the appropriate animal model should be selected on the basis of the objectives of the study. She also points out that results from animal systems must be compared with those from clinical evaluations.

P.L. Paggiaro and co-workers summarize the studies on the prognosis of occupational asthma, and conclude that a significant proportion of subjects continue to experience asthmatic symptoms and nonspecific airway hyperresponsiveness after cessation of work. In their review, they establish that the determinants of this unfavourable prognosis are long duration of exposure before the onset of asthma, long duration of symptoms before diagnosis, baseline airway obstruction, dual asthmatic reaction after specific inhalation challenge, and persistence of markers of airway inflammation in bronchoalveolar lavage fluid and bronchial biopsies.

K.M. Venables, in her review on prevention of occupational asthma, highlights the fact that the frequency with which asthma occurs in relation to other occupational lung diseases demands priority in designing preventive activities and research for this disease.

D.J. Hendrick reviews the current concepts concerning the management of occupational asthma. Occupational factors are likely to play a role in asthma in the population at large, therefore its successful management should contribute significantly to the overall control of asthma in the general population.

J.D. Dewitte and co-workers review the current strategies used to deal with the medicolegal and compensatory aspects of occupational asthma. They point out the necessity that diagnosis be proved by objective means, whenever feasible. They review the criteria of primary, secondary and tertiary prevention programmes. They conclude that the evaluation of permanent asthma and the awarding of disability compensation should be performed 2 yrs after exposure to the causal agent has ceased.

Occupational asthma is a potentially life-threatening condition and should be treated with the appropriate agents. It has to be clear that there is an absolute necessity for environmental controls in the workplaces, and for primary intervention in occupational asthma at an early stage in the course of the disease when reversibility can be achieved, at least in a proportion of affected workers.

\footnotetext{
Acknowledgements: Preparation of a series involves a significant amount of time and rational team-work. The author wishes to extend gratitude to clinical colleagues, research fellows, associates, nurses, and technicians who contributed to the research summarized in this series, and to thank the secretarial assistants, L. Tolin and G. Fulgeri; and finally, to mention the enthusiastic and most valuable work of C. Howarth for the editorial handling of the manuscripts. *Istituto di Medicina del Lavoro, Universita di Padova, Via Facciolati 71, I - 35127 Padova, Italy.
} 